\title{
Instability to a heterogeneous oscillatory state in randomly connected recurrent networks with delayed interactions
}

\author{
Célian Bimbard, ${ }^{1}$ Erwan Ledoux, ${ }^{2}$ and Srdjan Ostojic ${ }^{2}$ \\ ${ }^{1}$ Laboratoire des Systèmes Perceptifs, Équipe Audition, CNRS UMR 8248, École Normale Supérieure, Paris, France \\ ${ }^{2}$ Group for Neural Theory, Laboratoire de Neurosciences Cognitives, INSERM U960, \\ École Normale Supérieure-PSL Research University, Paris, France \\ (Received 26 May 2016; revised manuscript received 12 September 2016; published 12 December 2016)
}

\begin{abstract}
Oscillatory dynamics are ubiquitous in biological networks. Possible sources of oscillations are well understood in low-dimensional systems but have not been fully explored in high-dimensional networks. Here we study large networks consisting of randomly coupled rate units. We identify a type of bifurcation in which a continuous part of the eigenvalue spectrum of the linear stability matrix crosses the instability line at nonzero frequency. This bifurcation occurs when the interactions are delayed and partially antisymmetric and leads to a heterogeneous oscillatory state in which oscillations are apparent in the activity of individual units but not on the population-average level.
\end{abstract}

DOI: 10.1103/PhysRevE.94.062207

\section{INTRODUCTION}

Networks of interacting units are a fundamental model of many physical and biological systems, and understanding their dynamical repertoire is of outmost importance. Dynamical systems theory has examined extensively the dynamics of lowdimensional systems, and in particular bifurcations between different regimes [1]. These bifurcations typically occur when an eigenvalue of the system (or pair thereof) crosses an instability line, and enumerating the possible scenarios leads to an exhaustive taxonomy of dynamical behaviors for lowdimensional networks.

Many biological systems, in particular regulatory and neural networks, are, however, high dimensional as they consist of a large number of individual units, and the interactions between units are moreover strongly disordered. In contrast to low-dimensional systems, the dynamical repertoire of highdimensional networks of randomly interacting units has not been fully charted. In particular, within the context of randomly connected neural networks, a bifurcation has been identified, in which a continuum of eigenvalues loses stability, leading to a transition from constant, fixed-point activity to highly heterogeneous, chaotic activity [2]. This transition and its implications for neural computations have lately attracted a significant amount of attention [3-8].

In the bifurcation to chaotic activity described above, the continuous part of the spectrum that loses stability is centered around the real axis in the complex plane and leads to an instability at zero frequency. Here we show that an analogous but nonzero frequency instability can occur when interactions are delayed and partially antisymmetric. This type of bifurcation leads to a heterogeneous oscillatory state, in which different units oscillate at similar frequencies but random phases.

\section{NETWORK MODEL}

We investigated the dynamics of a network of $N$ randomly connected rate units [9] given by

$$
\frac{d x_{i}(t)}{d t}=-x_{i}(t)+\sum_{j=1}^{N} J_{i j} \phi\left[x_{j}(t-D)\right], \quad i=1 \ldots N
$$

where $x_{i}$ is the activity of unit $i, \phi(x)=\tanh (x)$ is the transfer function, and $D$ represents a delay in the interaction between the units. The elements of the interaction matrix $J$ are drawn from a Gaussian distribution of mean 0 and variance $g^{2} / N$. The symmetric elements $J_{i j}$ and $J_{j i}$ are correlated, and the degree of symmetry $\tau_{s}=\left\langle J_{i j} J_{j i}\right\rangle N / g^{2}$ is a parameter that we systematically varied. For $\tau_{s}=1$, the interaction matrix is fully symmetric, for $\tau_{s}=0$ it is fully asymmetric, and for $\tau_{s}=-1$ it is fully antisymmetric.

The network possesses a trivial fixed point for which the activity of all the units vanishes. We examined the stability of this fixed point as function of the strength of coupling $g$, the degree of symmetry in the interactions $\tau_{s}$, and the delay $D$. We considered heterogeneous perturbations in which the activity of each unit is perturbed away from the fixed point by an amount $\delta x_{i}(t)=\delta \tilde{x}_{i} e^{\lambda t}$, with $\lambda$ being an arbitrary complex number representing the decay of the perturbation.

By inserting the perturbation in Eq. (1) and linearizing around the fixed point we get

$$
e^{\lambda D}(1+\lambda) \delta \tilde{x}_{i}=\sum_{j=1}^{N} J_{i j} \delta \tilde{x}_{j}
$$

Diagonalizing the interaction matrix $J$ and projecting on the $k$ th eigenmode yields

$$
e^{\lambda_{k} D}\left(1+\lambda_{k}\right)=\mu_{k},
$$

where $\mu_{k}$ is the $k$ th eigenvalue of $J$. We therefore obtain $N$ independent characteristic equations that specify $N$ admissible values of the perturbation decay $\lambda_{k}, k=1 \ldots N$, one for each eigenvalue of $J$. For a given set of parameters $g, \tau_{s}$, and $D$, if any of these equations admits a solution with $\Re \lambda_{k}>0$, the corresponding perturbation is amplified and the fixed point is unstable.

In the limit of large $N$, the eigenvalues $\mu_{k}$ of the matrix $J$ are uniformly distributed within an ellipse centered at the origin in the complex plane, with real and imaginary axes $g\left(1+\tau_{s}\right)$ and $g\left(1-\tau_{s}\right)$ respectively [10]. Solving Eq. (3) for $\lambda$ yields a mapping that transforms this ellipse into a different domain in the complex plane that contains the solutions $\lambda_{k}$ of Eq. (3) (Fig. 1). We will call $\mu$ domain the domain containing 
(a)

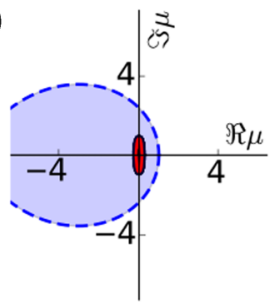

(c)

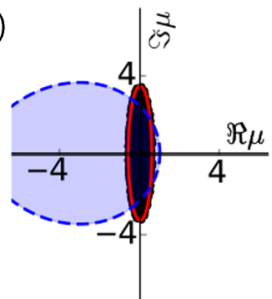

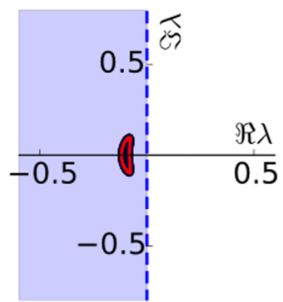

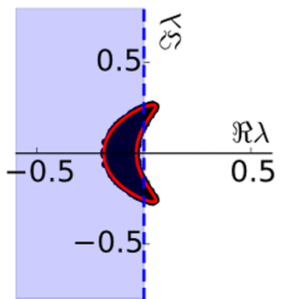

(b)

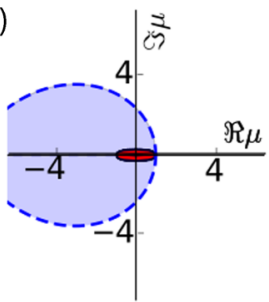

(d)

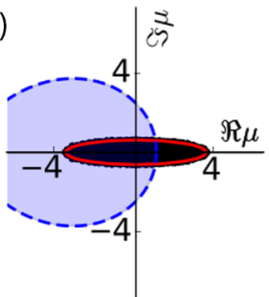

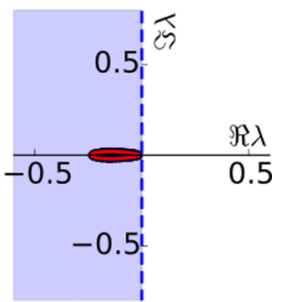

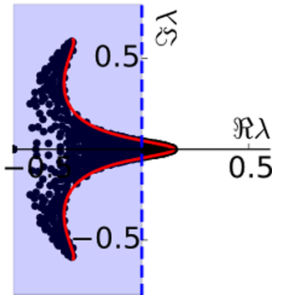

FIG. 1. Linear stability of the fixed point solution. (a)-(d) Left panel: Eigenvalues $\mu$ of a simulated interaction matrix (black dots), theoretically determined domain containing the eigenvalues (red ellipse) and stability domain (blue shaded region, dashed contour). Right panel: perturbation decay $\lambda$ associated to each eigenvalue $\mu$ (black dots), theoretical prediction of the $\lambda$ associated to the contour of the $\mu$ ellipse (red line) and stability domain (blue shaded region). Parameter values: (a) $\tau_{s}=-0.7$ and $g=0.2(N=1000, D=0.2)$, (b) same as in panel (a), with $\tau_{s}=0.7$ and $g=0.2$, (c) same as in panel (a), with $\tau_{s}=-0.7$ and $g=2.0$, and (d) same as in panel (a), with $\tau_{s}=0.7$ and $g=2.0$. All plotted quantities are dimensionless.

the eigenvalues $\mu_{k}$ of $J$, and $\lambda$ domain the domain containing the solutions $\lambda_{k}$ of Eq. (3). Note that the $\mu$ domain depends only on the values of $g$ and $\tau_{s}$, while the $\lambda$ domain depends also on the interaction delay $D$.

\section{PHASE DIAGRAM}

One approach for assessing the stability of the fixed point for a given set of parameters is to examine whether the resulting $\lambda$ domain intersects the line $\Re z=0$ in the complex plane. An alternative approach is to use the inverse mapping and determine the contour $\mu(z)$ in the $\mu$ plane that corresponds to $\Re \lambda=0$, and examine whether this contour intersects the $\mu$ domain containing the eigenvalues.

The two approaches are graphically illustrated in Fig. 1. For small values of the coupling $g$, the eigenvalues $\mu_{k}$ of $J$ have a small modulus, and all $\lambda_{k}$ solution of Eq. (3) have a negative real part: The synaptic coupling is too low to drive any instability of the fixed point. When the coupling $g$ is increased, the $\lambda$ domain crosses the imaginary axis [Figs. 1(c) and 1(d)]. In this case, the degree of symmetry of the matrix appears to play an important role for the shape of the $\lambda$ domain: In the antisymmetric case [Fig. 1(c)], values with $\Re \lambda>0$ have a nonzero imaginary part, whereas in the symmetric case [Fig. 1(d)], the solution $\lambda_{0}$ with largest real part has a vanishing imaginary part. We predict that these two cases are unstable, with the antisymmetric case possibly leading to an oscillatory behavior.

To systematically identify bifurcation points, we determined the coupling strength $g_{c}$ for which $\lambda=i \omega_{c}$ is a solution of a characteristic equation, Eq. (3). We first consider two simplifying cases, before describing the general solution.

For a symmetrically skewed matrix $\left(0<\tau_{s}<1\right)$, the ellipse that contains the eigenvalues of the matrix $J$ is elongated along the real axis. The eigenvalue $\mu_{0}$ with the largest modulus is therefore purely real and given by $\mu_{0}=g\left(1+\tau_{s}\right)$. The corresponding solution $\lambda_{0}$ of Eq. (3) is also real and vanishes when $\mu_{0}=1$, which yields the critical coupling $g_{c}=1 /\left(1+\tau_{s}\right)$. At this coupling, all other solutions of Eq. (3) have negative real parts, and hence we have a zero-frequency bifurcation.

For an antisymmetrically skewed matrix $\left(-1<\tau_{s}<0\right)$, the ellipse that contains the eigenvalues of the matrix $J$ is elongated along the imaginary axis, and the eigenvalue $\mu_{0}$ with the largest modulus is therefore purely imaginary and given by $\mu_{0}=i g\left(1-\tau_{s}\right)$. The corresponding solution $\lambda_{0}$ of Eq. (3) now has a nonzero imaginary part, and the solution $\lambda_{0}=i \omega_{c}$ is given by

$$
\begin{gathered}
\omega_{c}=\sqrt{\left[g\left(1-\tau_{s}\right)\right]^{2}-1} \\
D=\frac{1}{\sqrt{\left[g\left(1-\tau_{s}\right)\right]^{2}-1}} \arcsin \left(\frac{1}{g\left(1-\tau_{s}\right)}\right) .
\end{gathered}
$$

This solution leads to a bifurcation with a nonzero frequency.

More generally, the first eigenvalue that becomes unstable as the coupling $g$ is increased does not need to correspond to an extremity of the ellipse in the $\mu$ plane. To determine the location of the instability in the general case, we followed Refs. [11,12] and determined the contour $\mu(z)$ in the complex plane that is mapped to the line $\lambda=i \omega$ via Eq. (3). By parametrizing this contour in polar coordinates as $\mu(\theta)=$ $M(\theta) e^{i \theta}$, we have for $0<\theta<\pi$ (the contour is symmetric to the horizontal axis) [12]

$$
\begin{aligned}
M(\theta) & =\sqrt{1+\omega^{2}} \\
-\omega & =\tan (\omega D-\theta), \text { with } \theta-\frac{\pi}{2}<\omega D<\theta, \text { modulo } 2 \pi .
\end{aligned}
$$

This contour depends only on the value of the delay $D$ and defines a droplet-shaped stability boundary for the eigenvalues of $J$ (Fig. 1). The critical coupling $g_{c}$ is then determined 


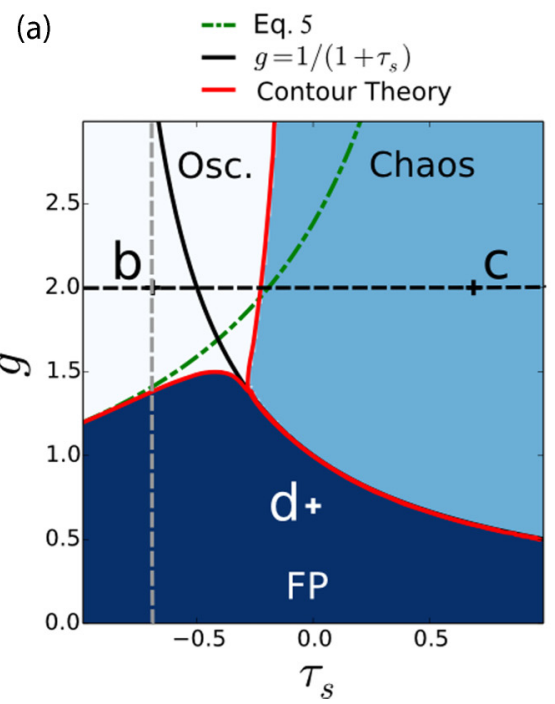

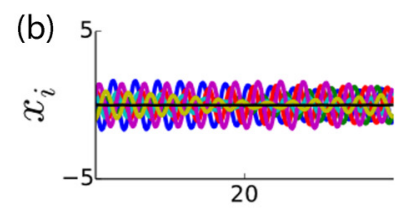
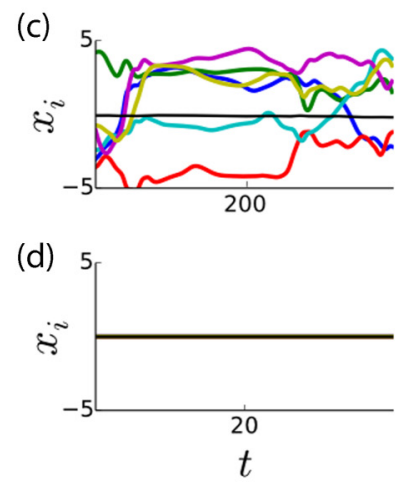

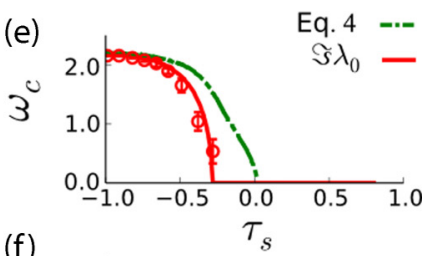

(f)
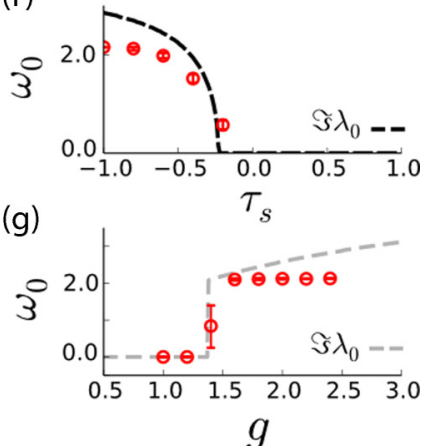

FIG. 2. Dynamical regimes of the network. (a) Bifurcation diagram. Dark blue region: fixed point (FP) is stable; light blue region: FP is unstable, with dominant eigenvalue real; white region: FP is unstable, dominant eigenvalue with a nonzero imaginary part. Red line (solid, trifold): exact theoretical prediction for the instability line. Black line (solid): approximation for $\tau_{s}>0$. Green line (dashed): approximation for $\tau_{s}<0$. (b)-(d) Illustrations of simulated dynamics in different regions of the bifurcation diagram $(N=1000, D=0.2)$. The panels show firing rates of ten neurons and the population average in black. Parameter values: (b) $\tau_{s}=-0.7$ and $g=2.0$, (c) $\tau_{s}=0.7$ and $g=2.0$, and (d) $\tau_{s}=0.7$ and $g=0.7$. (e) Bifurcation frequency $\omega_{c}$ computed along the instability line in panel (a), (red line), and approximation given by $\omega_{0}=\sqrt{\left[g\left(1-\tau_{s}\right)\right]^{2}-1}$ (dash-dotted green line). Simulation results are shown as red dots $(N=500, n=5$, mean \pm SEM). (f) Frequency of the dominant eigenvalue for constant $g$ as function of $\tau_{s}$ [along the black dashed line in panel (a)], compared to the frequency of oscillations in the simulated network (red dots). (g) As in panel (f), but for fixed $\tau_{s}$ and varying $g$ [along the gray dashed line in panel (a)]. All plotted quantities are dimensionless.

by the first intersection as coupling is increased between the elliptical $\mu$-domain containing the eigenvalues and the stability boundary defined by Eq. (6).

The full bifurcation diagram as function of symmetry $\tau_{s}$ and coupling $g$ for a fixed value of the delay $D$ is displayed in Fig. 2. For the symmetric case, $\tau_{s}>0$, the instability is given by the extremity of the ellipse on the real axis. It therefore occurs at zero frequency, and the critical coupling is given by $g_{c}=1 /\left(1+\tau_{s}\right)$. For couplings larger than $g_{c}$, the fixed point is unstable, and a chaotic, fluctuating state appears [2].

The zero-frequency, chaotic instability extends to the antisymmetric region until a critical value $\tau_{s}^{c}<0$ for which a nonzero frequency bifurcation appears. As $\tau_{s}$ is further decreased, the nonzero frequency instability is determined by eigenvalues closer to the extremity of the ellipse along the imaginary axis, so that the critical coupling and the frequency of the instability are well approximated by Eqs. (4) and (5).

The critical coupling $g_{c}$ and the frequency $\omega_{c}$ of the bifurcation strongly depend on the value of the delay $D$ in the interactions (Fig. 3). As the value of $D$ is increased from 0 , for a given value of $\tau_{s}$ both $g_{c}$ and $\omega_{c}$ progressively decrease. At the same time, $\tau_{s}^{c}$ increases, so that the oscillatory instability occupies an increasing portion of phase space. In the limit of infinitely long delays, $\tau_{s}^{c}$ tends to zero, so that the oscillatory instability extends until the boundary between antisymmetric and symmetric interactions.

\section{HETEROGENEOUS OSCILLATORY STATE}

In the region of the oscillatory instability, couplings larger than $g_{c}$ lead to a heterogeneous oscillatory state that we
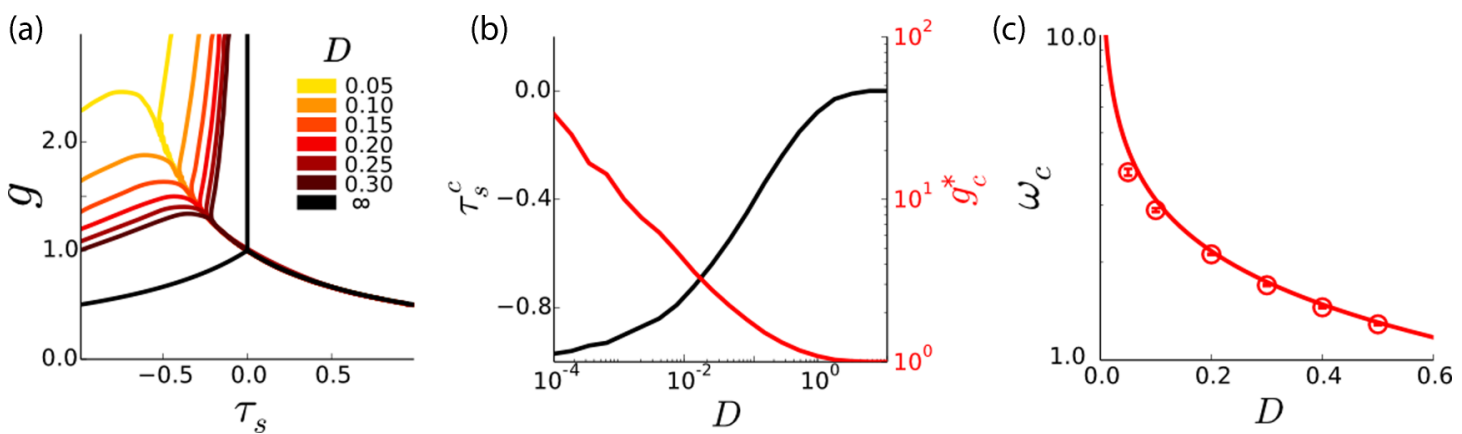

FIG. 3. Effects of interaction delay on network dynamics. (a) Bifurcation diagram for different values of interaction delay $D$. (b) Values of $\tau_{s}^{c}$ and corresponding coupling $g_{c}^{*}$ as function of delay. (c) Bifurcation frequency as a function of the normalized delay ( $\tau_{s}=-0.7, g$ adjusted). Full line: theoretical prediction; red dots: simulation results $(N=500, n=5$, mean $\pm \mathrm{SEM})$. All plotted quantities are dimensionless. 
(a)
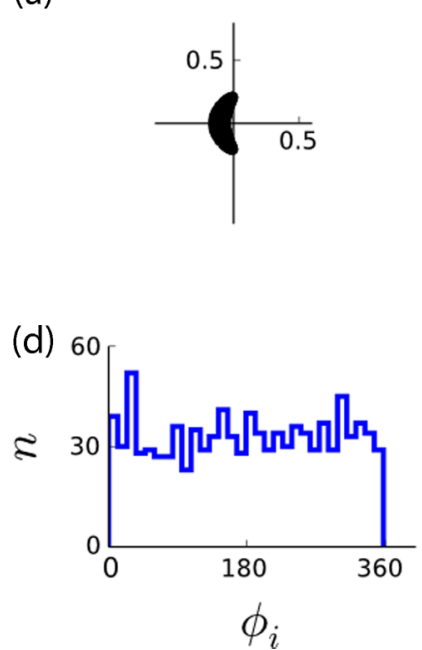
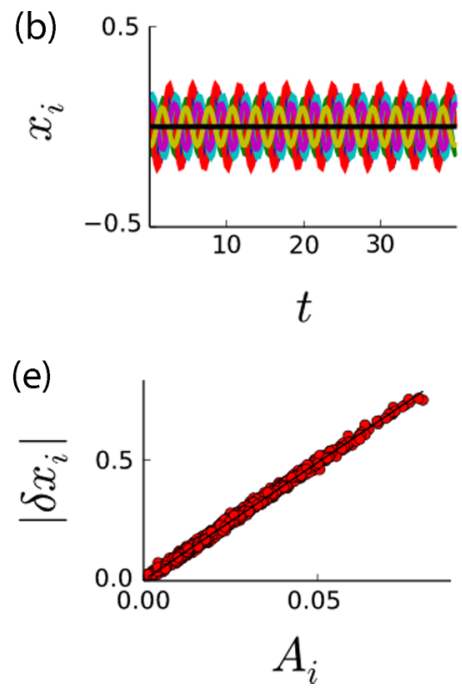
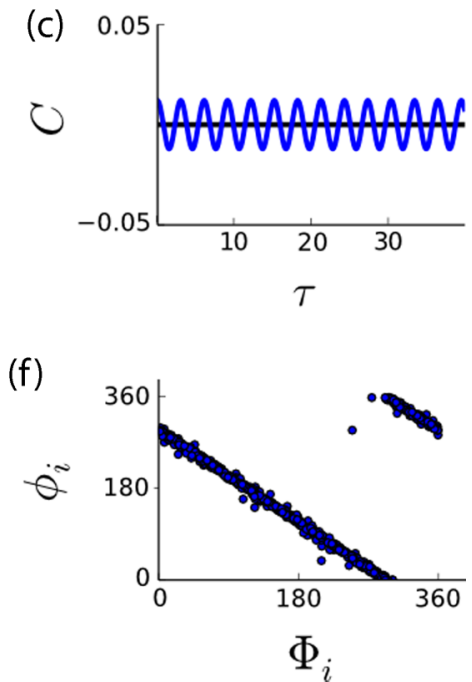

FIG. 4. Heterogeneous oscillations just above the instability $(g=1.4)$. Example simulation with only one $\lambda$ with a positive real part $(g=1.4)$. The connection matrix was found so that only one eigenvalue was unstable. The simulations were performed using $\tau_{s}=-0.7$ and $D=0.2(N=1000)$ : (a) Distribution of the computed $\lambda$. (b) Firing rate of 10 neurons superimposed. (c) Mean auto-correlogram of all neurons $C(\tau)$ (blue trace) and auto-correlogram of the mean population activity $K(\tau)$ (black). (d) Histograms of the phases of the firing rates of all neurons. (e) Correlation between the amplitudes of the firing rates and the module of each component of the principal eigenvector. (f) Correlation between the phases of the firing rates and the phase of each component of the principal eigenvector. All plotted quantities are dimensionless.

investigated using numerical simulations of Eq. (1). In this state, the different units appear to oscillate with essentially identical frequencies, but random phases and amplitudes (Fig. 4). To further characterize this state, we defined the phase of each unit as the phase of the first peak $\left(t_{\text {peak }}\right)$ with respect to an arbitrary reference common to all units $\left(t_{0}\right)$ :

$$
\phi_{i}=\left(t_{\text {peak }}^{i}-t_{0}\right) \omega_{0} \text { modulo } 2 \pi .
$$

Correspondingly, the amplitude of each unit was defined as the peak-to-peak amplitude $\left|\delta x_{i}\right|$. The phases of different units appear to be uniformly distributed over $[0,2 \pi]$ [Fig. 4(d)]. As a consequence the average population activity is constant in time, so that oscillations are apparent only on the level of individual units, but not on the population-average level. This was further confirmed by looking at the autocorrelation function of individual units, averaged over the network

$$
C(\tau)=\frac{1}{N} \sum_{i=1}^{N} \int_{-\infty}^{+\infty} x_{i}(t) x_{i}(t-\tau) d t,
$$

which showed clear oscillations. In contrast, the autocorrelation of network-averaged activity

$$
K(\tau)=\frac{1}{N^{2}} \int_{-\infty}^{+\infty} \sum_{i=1}^{N} x_{i}(t) \sum_{j=1}^{N} x_{j}(t-\tau) d t
$$

was flat.

The structure of the oscillatory activity can be understood from the linear analysis close to the instability. In the limit when a single eigenvalue crosses the instability [Figs. 4(a)4(f)], the oscillations are predicted to occur along a single mode:

$$
x_{i}(t) \sim e^{i \omega_{c} t} R_{i}^{(0)}+\text { c.c. }
$$

where $\left\{R_{i}^{(0)}\right\}_{i=1 \ldots N}$ is the right eigenvector of $J$ corresponding to the unstable eigenvalue $\lambda_{0}=i \omega_{c}$. Within this limit, all the units share the same oscillation frequency, and the distribution of amplitudes and phases of the different units is determined by the amplitudes and phases of the eigenvector $\left\{R_{i}^{(0)}=\right.$ $\left.A_{i} e^{i \Phi_{i}}\right\}_{i=1 \ldots N}$. A comparison with the simulations shows that close to the instability, the phases and amplitudes of individual units are indeed perfectly predicted by the phases and amplitudes of the unstable eigenvector [Figs. 4(e) and 4(f)]. The dynamics are therefore effectively one-dimensional, and the random amplitudes and phases of the oscillation are a direct consequence of the random distribution of the elements of the corresponding eigenvector ( $A_{i}$ and $\Phi_{i}$ respectively).

As the coupling is further increased above $g_{c}$, an increasing number of modes become unstable and the dynamics become more complex. The envelope of the oscillations starts to fluctuate [Fig. 5(b)], and the envelope of the autocorrelation function of individual units displays a slow decay [Fig. 5(c)]. This damping of oscillations may reveal underlying chaotic dynamics, an issue we have not explored further. The individual phases and amplitudes are not accurately predicted by the first eigenvector [Figs. 5(e) and 5(f)], yet the phases of individual units remain uniformly distributed on $[0,2 \pi]$ [Fig. 5(d)], so that the summed population activity is constant. The network therefore remains in a heterogeneous oscillatory state, in which individual units strongly oscillate, but this oscillation is not apparent on the population-average level.

To further quantify to dimensionality of the dynamics, we have computed projections of the activity on the left eigenvectors of the coupling matrix, defined as

$$
\frac{1}{T} \sum_{t=1}^{T}\left\langle L^{i} \mid x_{t}\right\rangle /\left\|x_{t}\right\|
$$


(a)

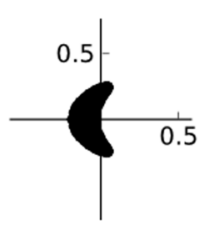

(d)

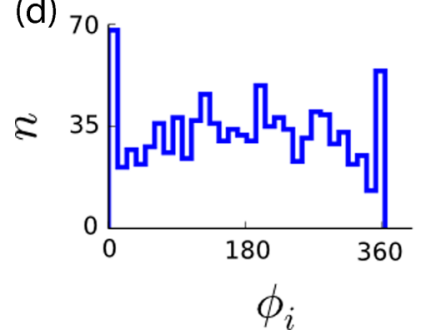

(b)

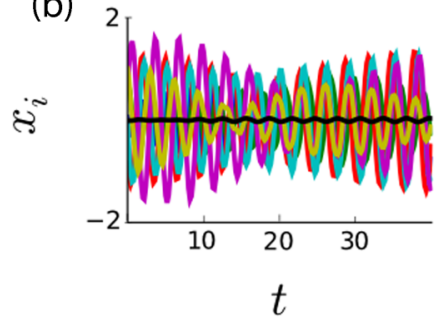

(e)

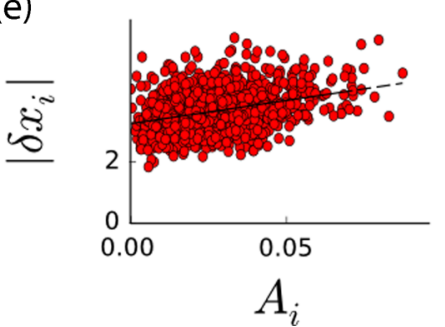

(c)

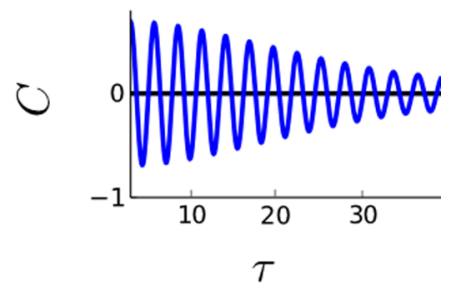

(f)

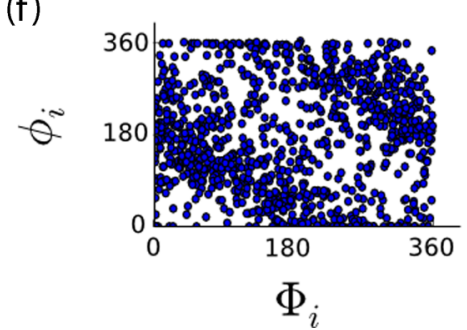

FIG. 5. Same as Fig. 4, but further above the instability $(g=2.0)$, where several eigenvalues have crossed the instability line.

with $T$ being the length of the time window used for all computations $\left(T=10^{7}\right)$. As the coupling is increased, the number of nonzero projections increases. This can be seen both when looking at all the sorted modes with a fixed coupling [Fig. 6(a)] or varying the coupling for a fixed eigenmode [Fig. 6(b)]. The oscillatory state explores more dimensions as the coupling is increased above the instability.

\section{DISCUSSION}

In summary, we have described a heterogeneous oscillatory state in network of randomly coupled units with delayed and partially antisymmetric interactions. This oscillatory state is governed by an undescribed type of bifurcation, in which a continuum of eigenvalues crosses the instability line at a nonzero frequency. In this state, individual units oscillate with random phases, so that the summed population activity remains constant in time.

From this perspective, the heterogeneous oscillatory state bears some similarity with the splay state, an asynchronous
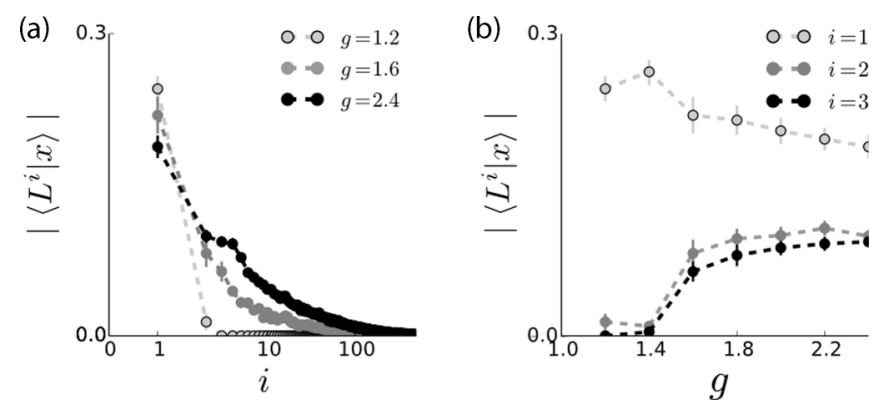

FIG. 6. Dimensionality of the dynamics above the oscillatory instability. (a) Projection of network activity $x$ on the left eigenvectors associated to the different eigenvalues, ordered by decreasing real part, for increasing coupling $g$ (conjugate eigenvectors are not shown). (b) Projection of $x$ on the left eigenvector associated to the first, second, or third eigenvalues as a function of $g$. Parameter values: $\tau_{s}=-0.7$ and $D=0.2, N=1000, n=30$, mean \pm SEM. All plotted quantities are dimensionless. regime in networks of interacting oscillators $[13,14]$. One important difference is that in the classical splay state the individual units oscillate even in the absence of coupling. In contrast, in the network considered here, the individual units are not intrinsic oscillators. In the absence of coupling, their activity is constant in time, and the oscillations appear only when the coupling increases beyond a critical value. Note also that while both the splay state and the heterogeneous oscillatory state are asynchronous, in the sense that the macroscopic activity displays no oscillations, they are distinct from other types of asynchronous states $[7,15,16]$ in which the individual units do not oscillate.

The instability described here is also related to the oscillatory Turing instability described in the context of random ecological networks [17]. That bifurcation is also generated by a continuous part of the eigenspectrum of the stability matrix and leads to heterogeneous oscillations in which different nodes in the network oscillate out of phase. The underlying mechanism is, however, different, as in Ref. [17] the interactions between nodes are symmetric and not delayed.

Following previous works, we considered here a simplified network with a Gaussian connectivity matrix and a transfer function symmetric around zero. Such a model does not implement basic biophysical constraints such as segregation between excitatory and inhibitory neurons or the requirement that neural activity is positive. The influence of such constraints on the zero-frequency instability to rate chaos have been investigated recently in a series of works $[7,8,18,19]$. These studies focused on the asymmetric case. Determining how the heterogeneous oscillatory state described here occurs in more realistic networks will require combining excitation-inhibition segregation with symmetry (or asymmetry) in the connections. This represents an additional challenge, as both ingredients influence the spectrum of the interaction matrix. Note that reciprocal connections between excitatory and inhibitory cells may represent a natural source of antisymmetric connectivity. Such connections have been in particular reported in the olfactory bulb and linked to the $\gamma$ oscillations occurring there [20]. It would be interesting to investigate further whether these oscillations are related to the phenomenon described here. 
Oscillatory activity is widespread in biological networks, and in particular in the brain [21]. Possible origins of these oscillations have mainly been conceptualized in terms of bifurcations in low-dimensional dynamical systems. Together with other recent results [22], this study suggest that highdimensional, disordered networks can lead to specific oscillatory mechanisms that should be taken into account when interpreting biological data.

\section{ACKNOWLEDGMENTS}

We are grateful to Vincent Hakim for discussions. This work was funded by the Programme Emergences of City of Paris, and the program "Investissements d'Avenir" launched by the French Government and implemented by the ANR, with the references ANR-10-LABX-0087 IEC and ANR-11-IDEX0001-02 PSL* Research University.
[1] S. H. Strogatz, Nonlinear dYnamics and Chaos: With Applications to Physics, Biology, Chemistry, and Engineering (Westview Press, Boulder, CO, 2014).

[2] H. Sompolinsky, A. Crisanti, and H. J. Sommers, Phys. Rev. Lett. 61, 259 (1988).

[3] D. Sussillo and L. F. Abbott, Neuron 63, 544 (2009).

[4] R. Laje and D. V. Buonomano, Nat. Neurosci. 16, 925 (2013).

[5] G. Wainrib and J. Touboul, Phys. Rev. Lett. 110, 118101 (2013).

[6] T. Toyoizumi and L. F. Abbott, Phys. Rev. E 84, 051908 (2011).

[7] S. Ostojic, Nat. Neurosci. 17, 594 (2014).

[8] J. Kadmon and H. Sompolinsky, Phys. Rev. X 5, 041030 (2015).

[9] P. Dayan and L. Abbott, Theoretical Neuroscience: Computational and Mathematical Modeling of Neural Systems (MIT Press, Cambridge, MA, 2001).

[10] H. J. Sommers, A. Crisanti, H. Sompolinsky, and Y. Stein, Phys. Rev. Lett. 60, 1895 (1988).

[11] J. Bélair, J. Dynamics Diff. Eq. 5, 607 (1993).
[12] C. M. Marcus and R. M. Westervelt, Phys. Rev. A 39, 347 (1989).

[13] L. F. Abbott and C. van Vreeswijk, Phys. Rev. E 48, 1483 (1993).

[14] L. Tattini, S. Olmi, and A. Torcini, Chaos 22, 023133 (2012).

[15] A. Renart, J. de la Rocha, P. Bartho, L. Hollender, N. Parga, A. Reyes, and K. D. Harris, Science 327, 587 (2010).

[16] N. Brunel, J. Comput. Neurosci. 8, 183 (2000).

[17] S. Hata, H. Nakao, and A. S. Mikhailov, Sci. Rep. 4, 3585 (2014).

[18] O. Harish and D. Hansel, PLoS Comput. Biol. 11, e1004266 (2015).

[19] F. Mastroguiseppe and S. Ostojic, arXiv:1605.04221.

[20] K. Rajan and L. F. Abbott, Phys. Rev. Lett. 97, 188104 (2006).

[21] G. Buzsáki, Rhythms of the Brain (Oxford University Press, Oxord, UK, 2006).

[22] L. C. Garcia del Molino, K. Pakdaman, J. Touboul, and G. Wainrib, Phys. Rev. E 88, 042824 (2013). 